\title{
A produção acadêmica sobre a rotulagem de alimentos no Brasil
}

\author{
Maria Clara Coelho Câmara, ${ }^{1}$ Carmem Luisa Cabral Marinho, ${ }^{1}$ \\ Maria Cristina Guilam ${ }^{1}$ e Ana Maria Cheble Bahia Braga ${ }^{1}$
}

Como citar Câmara MCC, Marinho CLC, Guilam MC, Braga AMCB. A produção acadêmica sobre a rotulagem de alimentos no Brasil. Rev Panam Salud Publica. 2008;23(1):52-58.

RESUMO Objetivo. Identificar e discutir a produção acadêmica (teses e dissertações) sobre a rotulagem de alimentos industrializados no Brasil.

Método. Foi pesquisado o portal da Coordenação de Aperfeiçoamento de Pessoal de Nível Superior (CAPES). Foram utilizados os descritores "rotulagem", "rotulagem nutricional" $e$ "rótulo de alimentos". A pesquisa abrangeu os anos de 1987 (primeiro ano do portal) a 2004. Resultados. Foram identificados 49 estudos que abordavam a temática proposta. Utilizando a técnica de análise de conteúdo foram identificados três tópicos centrais: adequação dos rótulos de produtos alimentícios à legislação específica $(57,2 \%)$, compreensão dos rótulos de alimentos pelos consumidores $(22,4 \%)$ e rotulagem de alimentos geneticamente modificados ou transgênicos $(20,4 \%)$.

Conclusões. A rotulagem de alimentos é um tema freqüente e abordado adequadamente pela produção acadêmica brasileira. A fiscalização ineficiente é apontada pela maioria dos estudos como principal fator para o descumprimento e a banalização das normas estabelecidas para a rotulagem de alimentos no Brasil.

Palavras-chave Rotulagem de alimentos, qualidade de produtos para o consumidor, Brasil.

\begin{abstract}
A legislação brasileira define rótulo como toda inscrição, legenda ou imagem, ou toda matéria descritiva ou gráfica, escrita, impressa, estampada, gravada, gravada em relevo ou litografada ou colada sobre a embalagem do alimento (1). Tais informações destinamse a identificar a origem, a composição

\footnotetext{
1 Fundação Oswaldo Cruz (Fiocruz), Centro de Estudos da Saúde do Trabalhador e Ecologia Humana (CESTEH). Enviar correspondência para Maria Clara Coelho Câmara no seguinte endereço: Rua Leopoldo Bulhões 1480, Prédio $1^{\circ}$ de Maio, CEP 21041-210, Rio de Janeiro, RJ, Brasil. Fone: +55-212598.2841; e-mail: maria.clara@ensp.fiocruz.br
}

e as características nutricionais dos produtos, permitindo o rastreamento dos mesmos, e constituindo-se, portanto, em elemento fundamental para a saúde pública. Cabe ressaltar que, no Brasil, as informações fornecidas através da rotulagem contemplam um direito assegurado pelo Código de Defesa do Consumidor que, em seu artigo $6^{\circ}$, determina que a informação sobre produtos e serviços deve ser clara e adequada e "com especificação correta de quantidade, características, composição, qualidade e preço, bem como sobre os riscos que apresentem" (2).
A rotulagem dos alimentos, ao orientar o consumidor sobre a qualidade e a quantidade dos constituintes nutricionais dos produtos, pode promover escolhas alimentares apropriadas (3-5), sendo indispensável, no entanto, a fidedignidade das informações. Tem sido observado que as falhas na legislação vigente no Brasil propiciam o repasse de informações incorretas, que podem gerar confusão, principalmente no que tange à informação nutricional complementar (INC) e às normas sobre alimentos para fins especiais (4). Entretanto, o 
acesso à informação correta sobre o conteúdo dos alimentos, por ser um elemento que impacta a adoção de práticas alimentares e estilos de vida saudáveis, configura-se, em seu conjunto, uma questão de segurança alimentar e nutricional (6).

A legislação brasileira de rotulagem tem por base as determinações do Codex Alimentarius, principal órgão internacional responsável pelo estabelecimento de normas sobre a segurança e a rotulagem de alimentos (7). O Codex Alimentarius tem como objetivo a proteção da saúde do consumidor, fixando, para tanto, diretrizes relativas ao plantio, à produção e à comercialização de alimentos, que devem servir de orientação para os cerca de 165 países membros, entre eles o Brasil.

No Brasil, a Agência Nacional de Vigilância Sanitária (ANVISA), ligada ao Ministério da Saúde, é responsável, entre outras atribuições, por fiscalizar a produção e a comercialização dos alimentos, além de normatizar a sua rotulagem. Embora a elaboração de leis para controle e vigilância de alimentos tenha tido início na década de 1950, somente com a criação da ANVISA, em 1999, a rotulagem nutricional tornouse obrigatória (8).

A primeira norma referente à rotulagem de alimentos no âmbito do $\mathrm{Mi}$ nistério da Saúde foi o decreto-lei $\mathrm{n}^{\circ} 986$ de 1969, que determina que "todo o alimento será exposto ao consumo ou entregue à venda depois de registrado no Ministério da Saúde". Tal decreto, ainda em vigor, estabelece a obrigatoriedade de informações como tipo de alimento, nome ou marca, nome do fabricante, local da fábrica, número de registro no Ministério da Saúde, indicação do emprego de aditivos intencionais, número de identificação da partida, lote, data de fabricação e indicação do peso ou volume, que devem constar de forma legível nas embalagens dos produtos (1). Desde então, diversas normas foram publicadas e revogadas. Atualmente, estão em vigor as portarias $27 \mathrm{e}$ 29 de 1998, referentes, respectivamente, à INC (9) e à rotulagem de ali- mentos para fins especiais, que "são os alimentos especialmente formulados, nos quais se introduzem modificações no conteúdo de nutrientes, adequados à utilização em dietas diferenciadas e/ou opcionais, atendendo às necessidades de pessoas em condições metabólicas e fisiológicas específicas" (10). Além dessas, as resoluções de diretoria colegiada (RDC) $n^{\circ} 259$ (11) e $n^{\circ} 40$ (12), de 2002, e a n 360 (13), de 2003, ainda em vigor, constituem-se as principais resoluções referentes à rotulagem dos alimentos industrializados, pois fixam os regulamentos técnicos para a rotulagem de alimentos embalados, a rotulagem nutricional e a obrigatoriedade da advertência "contém glúten". Esse arcabouço normativo é uma importante ferramenta para disponibilizar, de forma adequada e obrigatória, as informações relativas aos produtos disponíveis no mercado.

Dessa forma, o objetivo deste estudo foi identificar e discutir a produção acadêmica de teses e dissertações sobre a rotulagem de alimentos industrializados no Brasil.

\section{MATERIAIS E MÉTODOS}

Tendo como pressuposto que as dissertações e teses sobre rotulagem de alimentos configuram uma amostra que reflete o grau de importância dessa questão no País, utilizou-se o portal da Coordenação de Aperfeiçoamento de Pessoal de Nível Superior (CAPES), instituição responsável pela pós-graduação stricto sensu no Brasil, para obter esse material. Neste portal (http:/ / www.capes.gov.br/servicos/ bancoteses.html) encontram-se estudos produzidos em programas de mestrado (profissional e acadêmico) $(14,15)$ e doutorado (16). Definimos como "mestrado" o somatório das modalidades acadêmica e profissional.

As informações disponíveis, em formato de resumo, referem-se aos objetos de estudo, às metodologias utilizadas e aos principais resultados encontrados, elementos básicos, porém fundamentais, para a análise proposta por este estudo. A pesquisa foi efe- tuada utilizando-se os descritores "rotulagem", "rotulagem nutricional" e "rótulo de alimentos" e abrangeu todo o período disponível no portal no momento do estudo (anos de 1987 a 2004).

Para a análise das dissertações e teses, utilizou-se uma abordagem qualitativa, a técnica de análise de conteúdo, que visa, através de procedimentos sistemáticos e objetivos, a obter indicadores que possibilitem descrever o conteúdo dos textos, permitindo identificar as idéias centrais de cada um dos estudos encontrados (17).

\section{RESULTADOS}

Os descritores utilizados permitiram a identificação de 88 dissertações e teses para o período proposto. No entanto, 39 estudos $(44,3 \%)$ referiam-se a questões como arquitetura da informação, informática, rotulagem de produtos de limpeza e desodorantes e rotulagem ambiental. Apenas 49 estudos $(55,7 \%)$ abordavam efetivamente a temática de interesse de nosso estudo, sendo a maioria dissertações de mestrado elaboradas a partir da segunda metade da década de 1990 (18-66). As teses de doutorado corresponderam a apenas $8,2 \%$, o que provavelmente reflete o número menor de indivíduos que alcançam esse grau. Vale ressaltar que essa proporção de dissertações de mestrado para teses de doutorado reflete a relação entre os programas de pós-graduação reconhecidos pela CAPES, onde 2344 são cursos de mestrado e 1228 de doutorado (16).

A análise dos trabalhos identificados (18-66) revelou três tópicos como idéias centrais: em $57,2 \%$ ( 28 estudos) dos trabalhos, a idéia central foi a adequação dos rótulos de produtos alimentícios à legislação específica (18$22,25,30,33,34,35,37,38,40-42,45,48$, $49,51,53,54,56,58,61-63,65,66)$; em $22,4 \%$ (11 estudos), a compreensão dos rótulos de alimentos pelos consumidores $(23,26,28,29,31,32,36,44,55,57$, $59)$; e em $20,4 \%$ (10 estudos), a rotulagem de organismos geneticamente modificados (OGM)/transgênicos $(24,27$, $39,43,46,47,50,52,60,63)$. 


\section{Adequação do rótulo à legislação específica}

Como relatam as teses e dissertações investigadas, inúmeras normatizações têm sido estabelecidas com o objetivo de assegurar aos consumidores informações corretas e detalhadas sobre os produtos à venda no País. Das 28 dissertações e teses identificadas sobre este tópico, $71,4 \%$ analisaram, através de testes laboratoriais, a veracidade das informações presentes nos rótulos de alimentos com relação às determinações legais. Os demais estudos $(28,6 \%)$ apenas verificaram se as informações estavam de acordo com a legislação. Os principais produtos de análise foram: leite longa vida, azeite de oliva, produtos diet, queijos, carnes, margarinas, produtos importados, palmito e produtos enriquecidos. Os resultados, avaliados frente à legislação específica, apontaram um grande número de inadequações, principalmente em relação aos valores declarados nas informações nutricionais. Cabe destacar que tais inadequações permaneceram mesmo considerandose a tolerância permitida pela ANVISA de $20 \%$ com relação aos valores de nutrientes declarados no rótulo (13). Essa tolerância extremamente elevada, embora permitida pela ANVISA, desrespeita o Código de Defesa do Consumidor (2) vigente no País, uma vez que implica afirmar que os consumidores não têm acesso à quantidade exata dos nutrientes dos alimentos.

Um estudo analisou diferentes marcas de massas alimentícias e verificou que os valores encontrados em teste laboratorial para proteína, gordura, fibras alimentares, cálcio, ferro e sódio apresentavam discrepâncias quando comparados às informações contidas nos rótulos. Somente as quantidades de carboidrato e o valor calórico estavam de acordo com as informações declaradas (56). Uma análise semelhante foi realizada com pratos prontos para o consumo. O resultado demonstrou que os valores de carboidrato, proteína e ferro estavam superestimados, enquanto os percentuais de fibra alimentar e colesterol encontravam-se subestimados (40).
A avaliação de produtos para fins especiais (diet) (38) e de alimentos enriquecidos também apresentou resultados divergentes das informações contidas nos rótulos $(41,42)$. Um estudo que avaliou a rotulagem geral e nutricional do leite longa vida comercializado em Brasília, capital do País, verificou irregularidades em até $25 \%$ das marcas avaliadas quanto à rotulagem geral, enquanto na rotulagem nutricional essa porcentagem atingiu $57 \%$ dos produtos analisados (19). Estudos semelhantes, analisando queijos, lingüiças de frango e pernil, também observaram a mesma diferença entre a rotulagem geral e a nutricional $(48,54)$.

Com relação à rotulagem geral, os estudos analisados concluíram que, tanto nos produtos importados, como nos produtos nacionais, as inadequações eram as mesmas: ausência de informações sobre o número do lote, a data de fabricação, o prazo de validade, especificação dos corantes adicionados intencionalmente e informação sobre a presença de glúten $(45,63,65)$. Apenas a rotulagem de sopas desidratadas instantâneas e fórmulas para lactentes apresentam-se em conformidade com a legislação específica $(22,37)$.

Outra inadequação observada referese à rotulagem de produtos que contém glúten. Uma dissertação analisou produtos comerciais contendo aveia, verificando que três das cinco amostras de farinha de aveia avaliadas apresentavam glúten, substância que não deveria estar presente, contrariando as informações declaradas nos rótulos e na própria legislação (62). A não especificação dessa substância foi observada também por Picolotto (53) em análise de 98 tipos de alimentos naturalmente isentos. Esse autor observou que apenas 19 deles não continham tal proteína na sua composição.

\section{Compreensão dos rótulos de alimentos pelos consumidores}

Dos 11 estudos referentes a esse tópico, $45,4 \%$ (cinco estudos) abordavam as possíveis estratégias para influenciar o consumidor no momento da compra, através da mídia, da propaganda ou da publicidade. Os demais abordavam a influência que a rotulagem exerce sobre o consumidor na escolha dos alimentos.

Ao analisar as estratégias discursivas adotadas pela mídia quanto às práticas alimentares de emagrecimento de adolescentes brasileiros, Serra (57) identificou um discurso repleto de recursos de persuasão e sedução para o convencimento desses consumidores. Tal discurso, segundo a autora, pode influenciar as decisões, atitudes e comportamentos desses jovens em relação à prática alimentar, contrariando, muitas vezes, o conhecimento científico produzido pela área da nutrição. Segundo Gonsalves (36), a prática de incluir falsas informações, ou de ressaltar características intrínsecas ao produto como atributo exclusivo de uma determinada marca, visa a influenciar a estrutura de preferência do consumidor. $\mathrm{O}$ autor analisou iogurtes de diferentes marcas e identificou que $50 \%$ das amostras utilizavam informações tendenciosas.

De acordo com Coutinho (28), 43\% dos consumidores brasileiros, no ato da compra dos alimentos, buscam nas embalagens informações sobre os benefícios para a saúde. Muitos entrevistados afirmaram acreditar na capacidade de prevenção e controle que a alimentação pode exercer sobre doenças como o câncer, a hipertensão arterial, a obesidade e as doenças do coração. Outro estudo, realizado com freqüentadores de supermercados, concluiu que $61 \%$ dos entrevistados liam os rótulos dos produtos que compravam; porém, a autora ressaltou que tal conduta referia-se, particularmente, àqueles consumidores com problemas de saúde ou de classe social mais elevada (44).

Por outro lado, vários estudos identificaram a dificuldade do consumidor de compreender as informações disponibilizadas nos rótulos dos alimentos por não serem claras. Além disso, muitos consumidores não conheciam a rotulagem nutricional, e alguns não demonstraram interesse por esses dados $(32,43,44,59)$. 
Fatores como o tipo da embalagem e a sua repercussão sobre o ambiente também influenciaram a intenção de compra dos consumidores no Brasil $(26,29)$. A ilustração, o preço e a marca foram aspectos que influenciaram significativamente a intenção de compra. Com relação ao ambiente e à influência no processo de compra dos alimentos, observou-se que o atributo de produto "ambientalmente correto" esteve associado a alimentos cultivados sem agrotóxicos.

A leitura dos rótulos dos alimentos não implica, necessariamente, em intenção de compra por parte do consumidor, como demonstrou Silva (59). Nessa dissertação, apenas 23,6\% dos entrevistados modificaram os seus hábitos alimentares face às informações presentes nos rótulos, enquanto a maioria $(62,2 \%)$ afirmou desconhecer a rotulagem nutricional. O estudo concluiu que a influência da rotulagem nutricional sobre o consumidor ainda é incipiente, e que é necessário implementar ações educativas que esclareçam aos indivíduos a importância de considerar as informações nutricionais como um elemento determinante para a compra e o consumo dos alimentos.

\section{Rotulagem de transgênicos}

Os estudos referentes aos transgênicos mostraram a preocupação com a falta de cumprimento da legislação para a rotulagem dos produtos formulados a partir dos OGM $(23,24,27,39$, $46,50,52,64)$. Tais estudos enfatizaram a rotulagem dos transgênicos como um direito previsto no Código de Defesa do Consumidor (2), bem como uma oportunidade de controle da biotecnologia.

Os possíveis riscos decorrentes da utilização dos transgênicos foram tema de um estudo que analisou a relação do comércio internacional com o protocolo de biossegurança (documento resultante da Convenção de Diversidade Biológica, de 1995, que, entre outras determinações, reafirma a abordagem da precaução) (67), assim como as implicações da adesão ao princípio da precaução (uma ferramenta utilizada quando não é possível efetuar a avaliação científica do risco dos OGM, servindo para impedir ações que possam causar danos à saúde e ao meio ambiente) (67) e a necessidade de uma nova abordagem por parte do governo brasileiro sobre os OGM. Segundo a autora, a análise dos métodos participativos e deliberativos sobre esse tema é fundamental para a tomada de novas decisões a respeito dos transgênicos (47).

A atuação da sociedade civil, através das organizações não-governamentais, instaurando um forte debate público em torno da adoção dos transgênicos no Brasil, motivou outro estudo, que conclui que o novo cenário, incluindo os direitos de propriedade industrial da nova tecnologia, favorece as corporações transnacionais (60). É possível afirmar que, no Brasil, a sociedade civil organizada, no que se refere aos transgênicos, desempenhou o papel que caberia ao Estado, liderando estratégias e ações de cumprimento da decisão judicial que proibia o plantio e a comercialização dos transgênicos no País (67).

\section{DISCUSSÃO}

Em consonância com os estudos analisados neste trabalho, Araújo e Araújo (4) apontam que é significativo o número de produtos para fins especiais (tipo diet) que não seguem as normas de rotulagem, concluindo que é necessário maior rigor da ANVISA principalmente no caso desses alimentos, que têm uma função importante para o controle de doenças como o diabetes, uma doença crônico-degenerativa que implica em restrição a açúcares. Da mesma forma, a inadequação acerca da rotulagem de produtos que contêm glúten, relatada em duas teses $(53,62)$, é preocupante, já que a presença do glúten é prejudicial para portadores da doença celíaca, que consiste em uma intolerância permanente a essa proteína do trigo.

Alguns autores consideram que a rotulagem de alimentos no Brasil está muito aquém do padrão praticado na América do Norte e na Europa (67). Contudo, Celeste (68) afirma que, como no Brasil, as legislações do Mercosul, Reino Unido e União Européia também não exigem a descrição das recomendações nutricionais, um fator que considera essencial para que o consumidor tenha um parâmetro de comparação e possa, assim, escolher os produtos adequados para balancear a sua dieta. Essa autora considera ainda que a legislação brasileira sobre rotulagem deveria impedir a utilização de expressões e palavras em inglês e exigir a presença do percentual de energia fornecido pelos macronutrientes.

Gonsalves (36) observou outro caso para o qual o Brasil possui legislação, que, no entanto, não é cumprida: o uso de informações tendenciosas, contrariando a RDC $\mathrm{n}^{\circ} 259$, de 2002, que proíbe a utilização de qualquer tipo de expressão ou figura que possa levar o consumidor a engano (11). Dessa forma, parece evidente que, em muitos casos, embora exista a legislação, ela não é cumprida nem suficientemente fiscalizada.

A rotulagem dos OGM é um exemplo interessante levantado pelos artigos analisados. A rotulagem desses alimentos vem sendo, ao longo dos últimos anos, objeto de acirradas discussões. O foco da polêmica reside na argumentação de que a alteração nos rótulos para identificar os OGM representaria um acréscimo nos custos do produto, resultante da reformulação exigida para a inclusão do símbolo dos transgênicos ou do aumento físico do rótulo para acomodar tal informação. Além disso, foi levantada a possibilidade de que a inclusão de tal informação provocaria na população uma reação antecipadamente preconceituosa. Alega-se ainda que, do ponto de vista da equivalência substancial, a obrigatoriedade da segregação entre os alimentos transgênicos e os convencionais seria dispensável. Atualmente, no entanto, o processo de segregação é reconhecido como inevitável, diante do possível descrédito internacional motivado pela dificuldade em distinguir o grão convencional do transgê- 
nico (67). Assim, para as empresas processadoras, a rotulagem deveria representar um instrumento para esclarecer a origem do grão utilizado no caso do surgimento de problemas de saúde pública. A pressão da sociedade para que fossem estabelecidas e cumpridas as normas relativas aos transgênicos resultou no decreto $\mathrm{n}^{\circ} 3871$ (69), que determina que "os alimentos embalados, destinados ao consumo humano, que contenham ou sejam produzidos com organismo geneticamente modificado, com presença acima do limite de quatro por cento do produto, deverão conter informação nesse sentido em seus rótulos, sem prejuízo do cumprimento da legislação de biossegurança e da legislação aplicável aos alimentos em geral ou de outras normas complementares dos respectivos órgãos reguladores e fiscalizadores competentes".

\section{CONSIDERAÇÕES FINAIS}

Ao longo deste estudo, tentamos pesar a relevância da rotulagem de alimentos industrializados a partir da análise da produção acadêmica expressa em dissertações e teses brasileiras. Procuramos extrair as principais questões que, em linhas gerais, configuram a situação atual dessa problemática no País, e identificamos a inadequação na rotulagem de alimentos, a influência da rotulagem sobre os consumidores e a polêmica em torno da rotulagem dos OGMs como focos centrais.

Uma das principais conclusões deste estudo é que existem muitas inade- quações presentes nos rótulos de alimentos, particularmente quanto às informações nutricionais, mas que essas inadequações resultam menos da ausência de leis do que da falta de fiscalização. É inegável a contribuição do conjunto de normas e leis à rotulagem no Brasil; no entanto, é necessário transformar a intenção em ação, ou seja, a aplicação da legislação precisa ser alvo de uma efetiva fiscalização. $\mathrm{O}$ direito do consumidor a escolhas alimentares mais adequadas à sua saúde, ou estilo de vida, não está assegurado apenas pela existência de um amplo arcabouço legal, necessitando de vigilância permanente. Assim, instrumentalizar o consumidor para que ele próprio possa exercer a vigilância sobre o que compra e, sobretudo, consome, pode constituir-se em estratégia inicial.

\section{REFERÊNCIAS}

1. Brasil, Ministério da Marinha de Guerra do Exército e da Aeronáutica Militar. Decreto-lei $\mathrm{n}^{\circ}$ 986/69 sobre rotulagem de alimentos embalados. Brasília: Ministério da Marinha de Guerra do Exército e da Aeronáutica Militar 1969. Disponível em: http://e-legis.bvs.br/ leisref/public/showAct.php?id=1471. Acessado em dezembro de 2007.

2. Brasil, Ministério da Justiça. Código de Defesa do Consumidor (CDC). Lei $n^{\circ} 8$ 078/90 de 11 de setembro de 1990. Disponível em http:/ / www.planalto.gov.br/ccivil/LEIS/L8078. htm. Acessado em setembro de 2006.

3. Mantoanelli G, Colucci ACA, Philippi ST, Fisberg RM, Latterza AR, Cruz ATR. Avaliação de rótulos e embalagens de alimentos infantis: bebidas lácteas, iogurte e queijo tipo "Petit Suisse". Hig Alimentar. 1999;13(60):21-8.

4. Araujo ACMF, Araujo WMC. Adequação à legislação vigente, da rotulagem de alimentos para fins especiais dos grupos alimentos para dietas com restrição de carboidrato e alimentos para dieta de ingestão controlada de açúcares. Hig Alimentar. 2001;15(82):52-70.

5. Coutinho JG, Recine E. Experiências internacionais de regulamentação das alegações de saúde em rótulos de alimentos. Rev Panam Salud Publica/Pan Am J Public Health. 2007; 22(6):432-7.

6. Valente FLSV. Do combate à fome à segurança alimentar e nutricional: o direito à alimentação adequada. Em: Valente FLSV, org. Direito humano à alimentação: desafios e conquistas. São Paulo: Cortez; 2002. Pp. 37-70.

7. Kimbrell E. What is codex alimentarius? AgBioForum. 2000;3(4):197-202. Disponível em: http://www.agbioforum.org./v3n4/ v3n4a03-kimbrell.htm. Acessado em junho de 2003.

8. Paiva AJ, Henriques P. Adequação da rotulagem de alimentos diet e light ante a legislação específica. Rev Baiana Saude Publica. 2005; 19(Supl 1):39-48.

9. Brasil, Ministério da Saúde, Agência Nacional de Vigilância Sanitária. Informação nutricional complementar. Portaria ${ }^{\circ} 27$ de 13 de janeiro de 1998. Brasília: Ministério da Saúde 1998. Disponível em: http://www.anvisa. gov.br/alimentos/legis/especifica/rotuali. $\mathrm{htm}$. Acessado em maio de 2006.

10. Brasil, Ministério da Saúde, Agência Nacional de Vigilância Sanitária. Alimentos para fins especiais. Portaria $n^{\circ} 29$ de 13 de janeiro de 1998. Brasília: Ministério da saúde; 1998. Disponível em: http://www.anvisa.gov.br/ alimentos/legis/especifica/rotuali.htm. Acessado em maio de 2006

11. Brasil, Ministério da Saúde, Agência Nacional de Vigilância Sanitária. Rotulagem geral de alimentos embalados. Resolução RDC ${ }^{\circ} 259$ de 20 de setembro de 2002. Brasília: Ministério da saúde; 2002. Disponível em: http://www. anvisa.gov.br/alimentos/legis/especifica/ rotuali.htm . Acessado em maio de 2006.

12. Brasil, Ministério da Saúde, Agência Nacional de Vigilância Sanitária. Determinação de glúten dos alimentos. Resolução RDC $\mathrm{n}^{\circ} 40$ de 8 de fevereiro de 2002. Brasília: Ministério da Saúde; 2002. Disponível em: http://www. anvisa.gov.br/alimentos/legis/especifica/ rotuali.htm . Acessado em maio de 2006.

13. Brasil, Ministério da Saúde, Agência Nacional de Vigilância Sanitária. Informação nutricional. Resolução RDC n 360 de 23 de dezembro de 2003. Brasília: Ministério da saúde; 2003. Disponível em: http://www.anvisa.gov.br/ alimentos/legis/especifica/rotuali.htm. Acessado em 21 maio de 2006.

14. Moreira MA. O mestrado (profissional) em ensino. RBPG. 2004;1(jul):131-42.

15. Andrade C, D'Ávila C, Oliveira F. Um olhar sobre a práxis pedagógica do mestrado profissional em administração da Universidade Federal da Bahia. RBPG. 2004;1(2):81-96. Disponível em: http://www2.capes.gov.br/ rbpg/portal/conteudo/81_96_olhar_sobre praxi_pedagogica_mestrado_profissional.pdf. Acessado em dezembro de 2007.

16. Coordenação de Aperfeiçoamento de Pessoal de Nível Superior (CAPES). Histórico. 2006. Disponível em: http://www.capes.gov.br/ servicos/bancoteses.html. Acessado em 12 de setembro de 2006.

17. Bardin L. Análise de conteúdo. Lisboa: Edições 70; 1995

18. Barrera SM. Análise quantitativa e qualitativa da rotulagem de produtos à base de creatina para praticantes de atividades físicas [dissertação]. São Paulo: Universidade de São Paulo; 2004.

19. Barros DLG. Qualidade de leite UHT comercializado em Brasília-DF [dissertação]. Brasília: Universidade de Brasília; 2003.

20. Barroqueira ESB. Efeito do mercado do babaçu sobre a produção de anticorpos autoreativos [dissertação]. Maranhão; Universidade Federal do Maranhão; 2001.

21. Cardoso L. Qualidade dos alimentos comercializados no Distrito Federal-1997/2001 [dissertação]. Brasília: Universidade de Brasília; 2002. 
22. Carmona SG. Determinação por análise química de macronutrientes, energia e ferro em formulas lácteas para lactentes sadios no primeiro ano de vida [dissertação]. São Paulo: Universidade de São Paulo; 2003.

23. Meira DES. A obrigatoriedade da rotulagem no comércio de alimentos transgênicos [dissertação]. São Paulo: Pontifícia Universidade Católica de São Paulo; 2004.

24. Carvalho TDA. Uma análise jurídica da inserção dos organismos geneticamente modificados no Brasil: uma análise sob o ponto de vista dos princípios constitucionais [dissertação]. Belo Horizonte: Universidade Federal de Minas Gerais; 2003.

25. Clemente AL. Caracterização físico-química, perfil de ácidos graxo e ácido linoléico conjugado (CLA) em margarinas comerciais [dissertação]. Lavras: Universidade Federal de Lavras; 2004

26. Costa MC. Tecnologias não-convencionais e o impacto no comportamento do consumidor [dissertação]. Rio de Janeiro: Universidade Federal do Rio de Janeiro; 1999.

27. Solimene RCC. Rotulagem de alimentos transgênicos: responsabilidade da administração por deficiência na fiscalização [dissertação]. São Paulo: Pontifícia Universidade Católica de São Paulo; 2004

28. Coutinho JG. Estabelecimento de alegação de saúde nos rótulos de alimentos e bebidas embalados [dissertação]. Brasília: Universidade de Brasília; 2004.

29. Cuperschimid NRM. Atitude em relação ao meio ambiente e sua influência no processo de compra de alimentos em Curitiba [dissertação]. Curitiba: Universidade Federal do Paraná; 1999

30. Damboriarena E. Certificação e rotulagem como fator de competitividade na cadeia de hortigranjeiros no estado do RS: um estudo de caso-CEASA/RS [dissertação]. Porto Alegre: Universidade Federal do Rio Grande do Sul; 2001.

31. Dias M dos S. Fatores associados com a perda de peso corporal em hipertensos obesos ou com sobrepeso [dissertação]. Rio de Janeiro: Universidade Federal do Rio de Janeiro; 2003

32. Ferraz RG. Comportamento do consumidor frente à informação nutricional em rotulagem de produtos alimentícios [dissertação] Viçosa: Universidade Federal de Viçosa; 2001.

33. Ferreira AB. Conhecendo melhor a rotulagem dos alimentos: uma análise crítica [dissertação]. São Paulo: Universidade de São Paulo; 2004.

34. Garcia T. Estabilidade das vitaminas A, C e E em gomas de gelatina [dissertação]. São Paulo: Universidade de São Paulo; 2003.

35. Gomes EC. Capim-Limão-Cymbopogon citratus (D.C) Stapf: Subsídios para melhoria de qualidade do cultivo, industrialização e comercialização dos estados do Paraná [tese]. Curitiba: Universidade Federal do Paraná; 2003.

36. Gonsalves AI. Marketing nutricional em rotulagem de iogurtes: uma avaliação clínica [dissertação]. São Paulo: Universidade de São Paulo; 1997.

37. Guimarães CP. Estimativa dos teores de fenilalanina em sopas desidratadas instantâneas, importância do nitrogênio de origem não protéica [dissertação]. São Paulo: Universidade de São Paulo; 2003.
38. Guimarães EAH. Alimentos para fins especiais: observância da legislação quanto a rotulagem de dietéticos em restrição de açúcares [dissertação]. Recife: Universidade Federal de Pernambuco; 1999

39. Leite MC. Rotulagem de alimentos transgênicos: uma demanda da sociedade [dissertação] Brasília: Universidade de Brasília; 2003.

40. Lima A. Rotulagem nutricional de pratos prontos para o consumo: análise da metodologia [dissertação]. Recife: Universidade Federal de Pernambuco; 2002.

41. Lima JAC. Contribuição ao estudo do ácido fólico em alimentos enriquecidos [dissertação]. Campinas: Universidade Estadual de Campinas; 2001.

42. Lira ML. Aplicação e comparação de metodologias analíticas para determinação de vitamina C por CLAE em alimentos enriquecidos [dissertação]. João Pessoa: Universidade Federal da Paraíba; 2001.

43. Mandon SNTG. Comportamento de consumo e desenvolvimento do protótipo de um alimento destinado à população adulta e idosa brasileira [tese]. Florianópolis: Universidade Federal de Santa Catarina; 2003.

44. Martins BR. Análise do hábito de leitura e entendimento/recepção das informações contidas em rótulos de produtos alimentícios embalados, pela população adulta freqüentadora de supermercados, no Município de Niterói/ RJ [dissertação]. Rio de Janeiro: Fundação Instituto Oswaldo Cruz; 2004.

45. Mascarenhas JM. O. Corantes em alimentos: perspectivas, usos e restrições [dissertação]. Viçosa: Universidade Federal de Viçosa; 1997.

46. Morais RJ. Segurança e rotulagem de alimentos. Abordagem do direito econômico [dissertação]. Belo Horizonte: Universidade Federal de Minas Gerais; 2003.

47. Oliva LC. Análise de riscos e biossegurança [dissertação]. Rio de Janeiro: Universidade Federal do Rio de Janeiro; 2004.

48. Oliveira MJ de. Quantificação de nitrito e nitrato, avaliação físico-química, análise de rotulagem e riscos químicos em lingüiças do tipo frescal comercializadas em Brasília/DF [dissertação]. Brasília: Universidade de Brasília; 2003.

49. Ornelas LSC. Qualidade dos alimentos comercializados no Distrito Federal [dissertação] Brasília: Universidade de Brasília; 2003.

50. Paz VC. Alimentos e biossegurança: o caso da soja transgênica [dissertação]. Porto Alegre: Universidade Estadual do Rio Grande do Sul; 2004.

51. Peixoto ERM. Estudo de alguns parâmetros utilizados na avaliação da qualidade e identidade do azeite de oliva: proposta para modificação da legislação brasileira [dissertação] Rio de Janeiro: Universidade Federal do Rio de Janeiro; 1997.

52. Piccarelli DF. A rotulagem dos alimentos transgênicos no Código de Defesa do Consumidor [dissertação]. São Paulo: Universidade de São Paulo; 2003.

53. Picolotto FMBB. Determinação do teor de glúten por ensaio imunoenzimático em alimentos industrializados [tese]. Campinas: Universidade Estadual de Campinas; 2002.

54. Rocha FA. Análise da gordura total e do perfil de ácidos graxos em queijos do tipo mussarela, prato e ricota e comparação dos resulta- dos experimentais com os teóricos [dissertação]. Brasília: Universidade de Brasília; 2004. 55. Rosa LCC. Corantes naturais em alimentos: preferência do consumidor e aplicação industrial [dissertação]. Viçosa: Universidade Federal de Viçosa; 2004.

56. Sauverbronn ALA. Análise laboratorial da composição de alimentos processados como contribuição ao estudo da rotulagem nutricional obrigatória de alimentos e bebidas embalados no Brasil [dissertação]. Rio de Janeiro: Fundação Instituto Oswaldo Cruz; 2003.

57. Serra GMA. Saúde e nutrição na adolescência: o discurso sobre dietas da Revista Capricho [dissertação]. Rio de Janeiro: Fundação Instituto Oswaldo Cruz; 2001.

58. Silva MT. Determinação de conservantes químicos em refrescos de guaraná (Paullinia cupana, Mart. Ducke) e mate (Illex paraguariensis, St. Hill) por cromatografia gasosa de alta resolução [dissertação]. Rio de Janeiro: Universidade Federal Rural do Rio de Janeiro; 1997.

59. Silva MZT. Influência da rotulagem nutricional sobre o consumidor [dissertação]. Recife: Universidade Federal de Pernambuco; 2003.

60. Sanchez SSS. Crítica e reação em rede: o debate sobre os transgênicos no Brasil [tese]. São Paulo: Universidade de São Paulo; 2004.

61. Souza GR. Implantação do controle estatístico de processos em empresas de bebidas [dissertação]. Porto Alegre: Universidade Federal do Rio Grande do Sul; 2002.

62. Vieira EL. Determinantes de glúten em cultivares brasileiros de aveia e produtos derivados [dissertação]. Florianópolis: Universidade Federal de Santa Catarina; 2001.

63. Viesba RA. Elaboração de um sistema de análise de perigo e pontos críticos de controle para indústria de palmito em conserva: estudo de caso [dissertação]. Curitiba: Universidade Federal do Paraná; 2002.

64. Villares e Silva LF. A rotulagem dos alimentos transgênicos no Brasil [dissertação] São Paulo: Universidade de São Paulo; 2004.

65. Villela SHM. Análise de rótulos de alimentos embalados importados comercializados no varejo da cidade de Campinas, SP [dissertação]. Campinas: Universidade Estadual de Campinas; 2003.

66. Yosino EY. Alimento funcional: musse de chocolate enriquecido com fruto oligossacarídeos presentes no suco concentrado de yacon (Polymnia sonchifolia) [dissertação]. São Paulo: Universidade de São Paulo; 2003.

67. Marinho CLC. O discurso polissêmico sobre plantas transgênicas no Brasil: estado da arte [tese]. Rio de Janeiro: Fundação Instituto Oswaldo Cruz; 2003.

68. Celeste RK. Análise comparativa da legislação sobre rótulo alimentício do Brasil, Mercosul, Reino Unido e União Européia. Rev Saude Publica. 2001;35(3):217-23.

69. Brasil, Ministério da Saúde, Agência Nacional de Vigilância Sanitária. Rotulagem de organismos geneticamente modificados. Decreto $\mathrm{n}^{0}$ 3871 de 18 de julho de 2001. Brasília: Ministério da saúde; 2001. Disponível em: http://www. planalto.gov.br/CCIVIL/decreto/2001/D3871. $\mathrm{htm}$. Acessado em novembro de 2007.

Manuscrito recebido em 6 de janeiro de 2007. Aceito em versão revisada em 23 de julho de 2007. 
ABSTRACT Objective. To review and discuss academic production (theses and dissertations) on the topic of labeling of prepackaged foods in Brazil.

Academic production on food

Method. A search of the database maintained by the Coordination for the Development of Higher Education Professionals (CAPES), one of the two Brazilian government research funding and support agencies, was conducted on the following keywords: "rotulagem" (labeling), "rotulagem nutricional" (food labeling) and "rótulo de alimentos" (food labels). The search covered the years 1987 (earliest year available) to 2004 .

Results. We identified 49 studies on this topic. Content analysis identified three major themes: the extent to which food labels meet specific legal requirements $(57.2 \%)$; the degree to which consumers understand the information on labels $(22.4 \%)$; and the labeling of transgenic or genetically-modified foods $(20.4 \%)$.

Conclusions. Food labeling is a frequent topic and is adequately covered by the Brazilian academic production. In most of the studies, ineffective law enforcement appears to be the main factor in the lack of compliance with and disrespect for the food labeling rules and regulations in Brazil.

Key words Food labeling, consumer product safety, Brazil. 